\title{
Can Inferior Vena Cava Diameter and Collapsibility Index Be a Predictor in Detecting Preoperative Intravascular Volume Change in Pediatric Patients?
}

\author{
Zeliha Cosgun ${ }^{1}$, Emine Dagistan ${ }^{1}$, Mehmet Cosgun $^{2}$, Hayrettin Ozturk ${ }^{3}$ \\ ${ }^{1}$ Department of Radiodiagnostics, Medical School, Bolu Abant Izzet Baysal University, Bolu, Turkey \\ ${ }^{2}$ Department of Cardiology, Medical School, Bolu Abant Izzet Baysal University, Bolu, Turkey \\ ${ }^{3}$ Department of Pediatric Surgery, Medical School, Bolu Abant Izzet Baysal University, Medical School, Bolu, Turkey
}

\begin{abstract}
Background: Inferior vena cava (IVC) ultrasound measurement is a reliable indicator used in the assessment of intravascular volume status. The aim of this study was to evaluate intravascular volume changes in pediatric patients by measuring the IVC diameter and collapsibility index (CI) in children whose oral feeding was restricted preoperatively. Material and Methods: From May 2018 to October 2018, a total of 55 pediatric patients who were scheduled for surgery were included in this prospective, observational, cohort study. Fasting and satiety IVC diameters and CIs of patients were determined by ultrasonographic evaluation twice: in the preoperative preliminary evaluation, when the patients were satiated, and before surgery, during a fasting period of 6-8 hours. Ultrasonographic data were recorded and compared between fasting and satiety periods. Results: In the grey scale (B-mode), mean IVC diameter was significantly higher when the patients were satiated, compared to the measurements made just before surgery during the fasting period. In the $\mathrm{M}$ mode, the mean IVC diameter was significantly higher only during the inspiratory phase when the patients were satiated, while during the expiratory phase it was detected to be statistically similar. Mean CI was significantly higher in the immediate preoperative period, compared to the assessment made when satiated. Conclusion: Preoperative ultrasound IVC diameter and CI measurement can be a practical and useful method for evaluating preoperative intravascular volume in children.
\end{abstract}

Keywords: IVC diameter, collapsibility index, preoperative intravascular volume, pediatric patients

\section{ARTICLE HISTORY}

Received: January 17, 2021

Accepted: March 7, 2021

\section{Hayrettin Ozturk}

Bolu Abant İzzet Baysal Uninversity

Gölköy Yerleşkesi 14030

Merkez/Bolu, Turkey

Tel: +90 37425346563220

E-mail: ozturkhayrettin@hotmail.com

\section{INTRODUCTION}

The evaluation of hydration status in children is of utmost importance. Acute overhydration can lead to acute pulmonary edema and hypertension, while chronic overhydration is a well-known cardiovascular morbidity and mortality factor in children., ${ }^{1,2}$ Therefore, the evaluation of fluid responsiveness (FR) is very important for the diagnostic and therapeutic management of critically ill pediatric patients, since hypo- or hypervolemia may contribute to poor clinical outcomes. ${ }^{3,4}$ 
Today, there are many complementary methods for the clinical evaluation of hydration status: cardiothoracic index based on chest $\mathrm{x}$-ray evaluation, ultrasound-assessed inferior vena cava (IVC) diameter, brain natriuretic peptide, bioimpedance spectroscopy, plasmatic volume variation monitoring, and lung ultrasound in recent times., IVC is a major vessel that directly reflects the right heart function with high specificity. In addition, IVC is not affected by the body's compensatory vasoconstrictor response to volume loss. Because of all this, it may reflect the body's fluid deficit earlier than other arterial parameters such as blood pressure, pulse rate, and aortic diameter. ${ }^{7}$ Although the evaluation of intravascular volume by central venous pressure (CVP) measurement is a valuable method, it is an invasive procedure. The diameter and shape of IVC may vary according to CVP and intravascular volume. Therefore, the sonographic measurement of IVC is an effective and noninvasive method that can be used to estimate CVP. ${ }^{8-10}$

Many studies have been conducted to determine the fluid deficit with the help of the IVC diameter and collapsibility index (CI) in the postpartum period, in trauma, cardiogenic-hypovolemic and septic shock patients. ${ }^{11-13}$ However, to our knowledge, there is no study evaluating preoperative intravascular volume with IVC diameter and CI in the pediatric patient group. Therefore, this study was designed to evaluate changes in IVC diameter and CI of the restriction of preoperative oral nutrition in pediatric patients.

\section{MATERIALS AND METHODS}

This was a prospective, observational, cohort study conducted at a tertiary referral center in Turkey. The study protocol was approved by the local ethics committee (decision number: 126, approval date: April 4, 2018). The study was conducted in accordance with the principles stated in the Declaration of Helsinki. Parents of all patients and the institution were informed about the study, and their written consents were obtained. The study population consisted of 55 patients ( 33 male/22 female) with ages between 2 and 12 years, who were admitted to the anesthesia outpatient clinic for preoperative evaluation and scheduled for pediatric urologic surgery such as circumcision, undescended testes, hypospadias, and ureteropelvic junction obstruction between May 2018 and October 2018. In order to measure the fasting and satiety IVC diameter and CI, all patients received ultrasonographic evaluation twice: during the preliminary preoperative assessment when they were satiated, and before surgery, after mandatory fasting. The fasting period ranged between 6 and 8 hours, and appropriate perioperative maintenance fluid was infused in all cases. Ultrasonographic data of patients were recorded and then comparatively analyzed. Patients below 2 years and above 12 years, those with a previously known chronic disease, and those who had suboptimal sonographic evaluation due to preoperative anxiety were excluded from the study.

\section{ULTRASONOGRAPHIC MEASUREMENTS}

A low-frequency convex probe $(3 \mathrm{MHz}$ ) with an Esaote MyLab Five device was used for evaluation. IVC measurements were obtained in B-mode from a subxiphoid transabdominal long axis in the supine or right lateral position approximately $2-3 \mathrm{~cm}$ inferior of the right atrial junction. M-mode measurements were performed as recommended
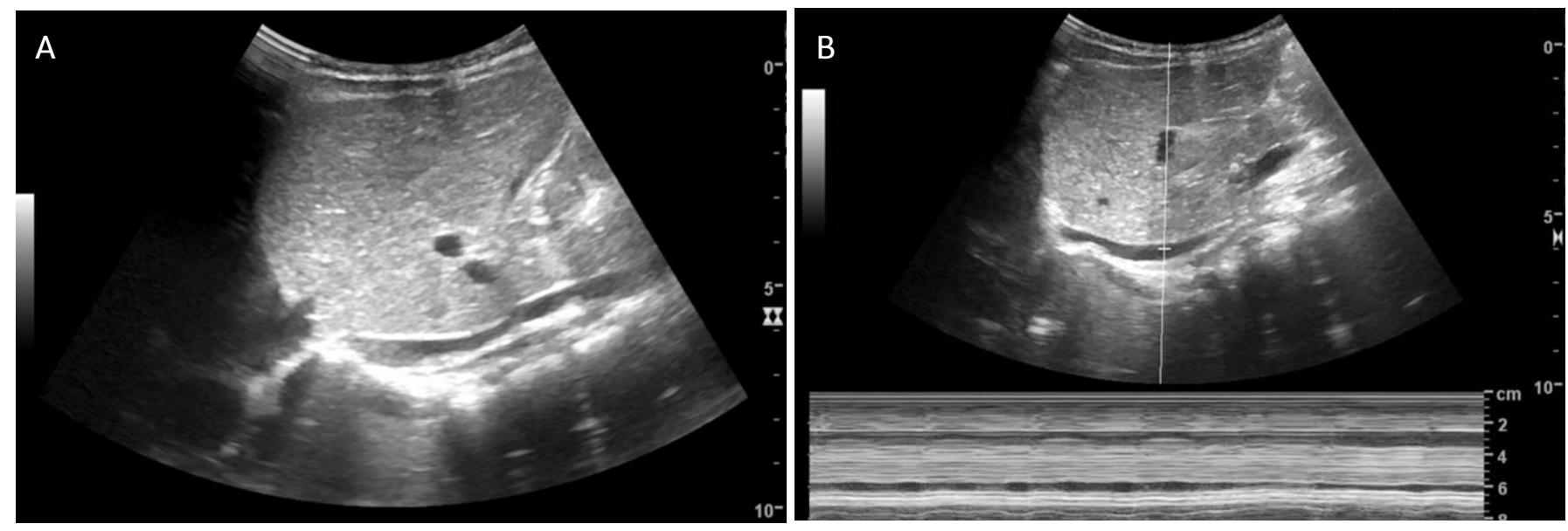

FIGURE 1. Ultrasound measurements of inferior vena cava (IVC) and collapsibility index (CI). A - Transabdominal long axis measurement; B - M-mode imaging from the transabdominal long axis. 
TABLE 1. Preoperative inferior vena cava (IVC) measurements in $\mathrm{B}$ - and $\mathrm{M}$-mode and collapse index $(\mathrm{Cl})$ during fasting and satiated periods

\begin{tabular}{lccc}
\hline Parameters & Fasting & Satiated & p value \\
\hline B-mode diameter $(\mathrm{mm})$ & $9.64 \pm 2.50$ & $10.33 \pm 2.33$ & 0.010 \\
M-mode expiratory diameter $(\mathrm{mm})$ & $9.80 \pm 2.76$ & $9.59 \pm 2.40$ & 0.468 \\
M-mode inspiratory diameter $(\mathrm{mm})$ & $5.84 \pm 1.95$ & $7.23 \pm 1.93$ & $<0.001$ \\
Collapsibility index & $0.40 \pm 0.10$ & $0.24 \pm 0.08$ & $<0.001$ \\
\hline
\end{tabular}

in the consensus document of the American and European Cardiologic Societies. ${ }^{-9}$ The CI was obtained by dividing the difference between the expiratory (eIVC) and inspiratory measurement of the IVC, divided by the eIVC [CI $=($ eIVC-IVC)/eIVC]. The IVC measurements at expiration and inspiration had to be measured during the same respiratory cycle (Figure 1). All ultrasonographic measurements were performed by the same experienced radiologist.

\section{STATISTICAL ANALYSIS}

The analysis was performed using the Statistical Package for Social Sciences 25.0 for Windows (SPSS Inc., Chicago Illinois, USA). The difference between the measurements was analyzed with paired samples $t$-tests, after assessing for normality. Data were presented as mean \pm standard deviation. The results were considered to be statistically significant at a level of $\mathrm{p}<0.05$.

\section{RESULTS}

When the measurements were made in the grey scale (Bmode), the mean IVC diameter was significantly higher when the patients were satiated, compared to the measurements made just before the surgery during the fasting period $(p=0.010)$. When the measurements were made in $M-$ mode, only the inspiratory mean VCI diameter was detected to be significantly higher when the patients were satiated, while the expiratory VCI diameter was not significantly different ( $p<0.001$ and $p=0.468$, respectively). Mean CI was significantly higher when measured during fasting in the preoperative period $(\mathrm{p}<0.001)$. The sonographic measurements of the patients are presented in Table 1.

\section{DISCUSSIONS}

In addition to the information obtained from physical examination and laboratory evaluation, invasive methods are frequently used to obtain more accurate and faster hemodynamic parameters. Among them, CVP is a com- monly used hemodynamic method. In addition, ultrasound examination, which is a noninvasive, practical and more economical method at the bedside and intensive care units, can also assist in assessing the fluid status of patients. ${ }^{14,15}$ Furthermore, ultrasound can evaluate the intravascular status by measuring the diameter of the IVC. ${ }^{16,17}$ This technique can evaluate the size and collapsibility of the IVC, similarly to the one used during cardiac ultrasound for the estimation of right atrial pressure (RAP) in non-acute care settings. Cyclic changes in thoracic pressure may be associated with a collapse of approximately $50 \%$ of the IVC diameter in cases without any other pathological conditions. ${ }^{18}$ The collapsibility of the IVC has been shown to provide accurate information for the noninvasive measurement of CVP in the follow-up of acute heart failure treatment and ongoing resuscitation. ${ }^{19,20}$ Zhang and Critchley suggested that preoperative ultrasonographic measurement of the IVC and CI had a good predictive value for hypotension after the induction of general anesthesia, with a sensitivity of $78.6 \%$ and a specificity of $91.7 \%$ at a cut-off value of $43 \% .{ }^{21}$ Tomohiro et al. found that the ultrasonographic measurement of the IVC diameter was a more accurate marker than vital parameters in predicting serious anemia after birth and identifying patients who will require blood transfusions. ${ }^{11}$ On the other hand, Sefidbakht et al..$^{12}$ and Yanagawa et al. ${ }^{13}$ showed that the rapid ultrasound performed for bedside IVC evaluation was more sensitive than blood pressure in assessing fluid loss in patients presenting in the emergency department for various types of trauma.

However, the IVC diameter and CI are mainly related to intrathoracic and intra-abdominal pressures and may be affected by increasing pathophysiological causes such as asthma or respiratory infection. ${ }^{22}$ Stawicki et al. have demonstrated that the CI strongly correlates with low $(<20 \%)$ and high $(>60 \%)$ CVP values and suggested that the closer the CI is to $0 \%$ or $100 \%$, the higher is the probability that the patient is either volume-overloaded or volume-depleted, respectively. ${ }^{23}$ Orso et al. ${ }^{24}$ have analyzed 26 studies that investigated the role of the caval index (IVC collapsibility or distensibility) and 5 studies on the IVC diameter. They observed an extreme heterogeneity of the included studies, and the meta-analysis highlighted that ultrasound evaluation of the diameter of the IVC and its respiratory variations is not a reliable method to predict fluid responsiveness.

The current study included cases that did not have any additional diseases, and were hemodynamically stable but were not given oral nutrition before the operation. In addition, these patients received parenteral maintenance 
fluid treatments during the time when their oral feeding was interrupted. Therefore, there was no condition in the study population that could have disrupted the hemodynamic balance such as hypovolemic shock. Hydration status was evaluated for patients exposed to a fasting period of 6-8 hours before the operation, and the results revealed that the IVC diameter and CI were high. Our results advocate for the importance and usefulness of preoperative evaluation of intravascular volume by assessment of the IVC diameter and CI in the pediatric age group. This noninvasive method based on ultrasound examination can offer valuable information regarding fluid deficit or overload, earlier than other arterial parameters such as blood pressure, pulse rate, and aortic diameter.?

Nevertheless, the present study has some limitations. First, this was a single-center assessment, and relatively few patients were included in the study. Therefore, a multicenter study is proposed to evaluate the cut-off point of the proposed parameters. Second, IVC collapsibility measurements can be affected by the craniocaudal movement of the diaphragm, causing two different areas of the IVC to be measured during inspiration and expiration, which may lead to underestimation of the IVC diameter and CI. Finally, the included cases were heterogeneous in terms of type of surgery. However, the inclusion of different types of surgery reflects clinical applicability in a more general pediatric population undergoing surgery.

\section{CONCLUSIONS}

In conclusion, the collapsibility index of the inferior vena cava, calculated by sonographic measurement of the inferior vena cava and $\mathrm{M}$-mode imaging, is an easily accessible and noninvasive method used in the pediatric patient group for assessment of intravascular fluid status in the preoperative period.

\section{CONFLICT OF INTEREST}

The authors declare no conflict of interest.

\section{REFERENCES}

1. Groothoff JW, Gruppen MP, Offringa M, et al. Mortality and causes of death of end-stage renal disease in children: a Dutch cohort study. Kidney Int. 2002;61:621-629. doi: 10.1046/j.1523-1755.2002.00156.x.

2. Wizemann V, Wabel $\mathrm{P}$, Chamney $\mathrm{P}$, et al. The mortality risk of overhydration in haemodialysis patients. Nephrol Dial Transplant. 2009;24:1574-1579. doi: 10.1093/ndt/gfn707.

3. Alsous F, Khamiees M, DeGirolamo A, Amoateng-Adjepong $\mathrm{Y}$, Manthous CA. Negative fluid balance predicts survival in patients with septic shock: a retrospective pilot study. Chest. 2000;117:1749-1754. doi: 10.1378/chest.117.6.1749.

4. Barbier C, Loubieres $Y$, Schmit C, et al. Respiratory changes in inferior vena cava diameter are helpful in predicting fluid responsiveness in ventilated septic patients. Intensive Care Med. 2004;30:1740-1746. doi: 10.1007/s00134-004-2259-8.

5. Arkouche W, Giaime P, Mercadal L. Les membres de la commission de dialyse de la Société de néphrologie [Fluid overload and arterial hypertension in hemodialysis patients]. Nephrol Ther. 2013;9:408-415. doi: 10.1016/j. nephro.2013.04.004.

6. Allinovi M, Saleem MA, Burgess O, Armstrong C, Hayes W. Finding covert fluid: methods for detecting volume overload in children on dialysis. Pediatr Nephrol Berl Ger. 2016;31:23272335. doi: 10.1007/s00467-016-3431-4.

7. Dipti A, Soucy Z, Surana A, Chandra S. Role of inferior vena cava diameter in assessment of volume status: a metaanalysis. Am J Emerg Med. 2012;30:1414-1419.e1. doi: 10.1016/j. ajem.2011.10.017.

8. Ciozda W, Kedan I, Kehl DW, Zimmer R, Khandwalla R, Kimchi A. The efficacy of sonographic measurement of inferior vena cava diameter as an estimate of central venous pressure. Cardiovasc Ultrasound. 2016;14:33. doi: 10.1186/s12947-0160076-1.

9. Babaie S, Behzad A, Mohammadpour M, Reisi M. A Comparison between the Bedside Sonographic Measurements of the Inferior Vena Cava Indices and the Central Venous Pressure While Assessing the Decreased Intravascular Volume in Children. Adv Biomed Res. 2018;7:97. doi: 10.4103/abr. abr_213_17.

10. Premkumar M, Rangegowda D, Kajal K, Khumuckham JS. Noninvasive estimation of intravascular volume status in cirrhosis by dynamic size and collapsibility indices of the inferior vena cava using bedside echocardiography. JGH Open. 2019;3:322-328. doi: 10.1002/jgh3.12166.

11. Oba T, Koyano M, Hasegawa $J$, et al. The inferior vena cava diameter is a useful ultrasound finding for predicting postpartum blood loss. J Matern Fetal Neonatal Med. 2019;32:3251-3254. doi: 10.1080/14767058.2018.1462321.

12. Sefidbakht S, Assadsangabi R, Abbasi HR, Nabavizadeh A. Sonographic measurement of the inferior vena cava as a predictor of shock in trauma patients. Emerg Radiol. 2007;14:181-185. doi: 10.1007/s10140-007-0602-4.

13. Yanagawa Y, Sakamoto T, Okada Y. Hypovolemic shock evaluated by sonographic measurement of the inferior vena cava during resuscitation in trauma patients. J Trauma. 2007;63:1245-1248. doi: 10.1097/TA.0b013e318068d72b.

14. Kastrup M, Markewitz A, Spies C, et al. Current practice of hemodynamic monitoring and vasopressor and inotropic therapy in post-operative cardiac surgery patients in Germany: results from a postal survey. Acta Anaesthesiol Scand. 2007;51:347-358. doi: 10.1111/j.13996576.2006.01190.x.

15. Ilyas A, Ishtiaq W, Assad S, et al. Correlation of IVC Diameter and Collapsibility Index With Central Venous Pressure in the Assessment of Intravascular Volume in Critically Ill Patients. Cureus. 2017;9:e1025. doi: 10.7759/cureus.1025.

16. Nagdev AD, Merchant RC, Tirado-Gonzalez A, Sisson CA, Murphy MC. Emergency department bedside ultrasonographic measurement of the caval index for noninvasive determination of low central venous pressure. Ann Emerg Med. 2010;55:290295. doi: 10.1016/j.annemergmed.2009.04.021. 
17. Fields JM, Lee PA, Jenq KY, Mark DG, Panebianco NL, Dean AJ. The interrater reliability of inferior vena cava ultrasound by bedside clinician sonographers in emergency department patients. Acad Emerg Med. 2011;18:98-101.

18. Sobczyk D, Nycz K, Andruszkiewicz P, Wierzbicki K, Stapor M. Ultrasonographic caval indices do not significantly contribute to predicting fluid responsiveness immediately after coronary artery bypass grafting when compared to passive leg raising. Cardiovasc Ultrasound. 2016;14:23. doi: 10.1186/s12947-0160065-4.

19. Thanakitcharu $P$, Charoenwut $M$, Siriwiwatanakul N. Inferior vena cava diameter and collapsibility index: a practical noninvasive evaluation of intravascular fluid volume in criticallyill patients. J Med Assoc Thai. 2013;96 Suppl 3:S14-S22.

20. Yavaşi Ö, Ünlüer EE, Kayayurt K, et al. Monitoring the response to treatment of acute heart failure patients by ultrasonographic inferior vena cava collapsibility index. Am J Emerg Med. 2014;32:403-407. doi: 10.1016/j.ajem.2013.12.046.

21. Zhang J, Critchley LA. Inferior vena cava ultrasonography before general anesthesia can predict hypotension after induction. Anesthesiology. 2016;124:580-589. doi: 10.1097/ ALN.0000000000001002.

22. Salama ER, Elkashlan M. Pre-operative ultrasonographic evaluation of inferior vena cava collapsibility index and caval aorta index as new predictors for hypotension after induction of spinal anaesthesia: A prospective observational study. Eur J Anaesthesiol. 2019;36:297-302. doi: 10.1097/ EJA.0000000000000956.

23. Stawicki SP, Braslow BM, Panebianco NL, et al. Intensivist use of hand-carried ultrasonography to measure IVC collapsibility in estimating intravascular volume status: correlations with CVP. J Am Coll Surg. 2009;209:55-61. doi: 10.1016/j. jamcollsurg.2009.02.062.

24. Orso D, Paoli I, Piani T, Cilenti FL, Cristiani L, Guglielmo N. Accuracy of Ultrasonographic Measurements of Inferior Vena Cava to Determine Fluid Responsiveness: A Systematic Review and Meta-Analysis. J Intensive Care Med. 2020;35:354-363. doi: 10.1177/0885066617752308. 